\title{
Evaluation of Setting Time and Solubility of Bio-ceramic Sealer Before and After Mixing with Prednisolone Powder: A Comparative In Vitro Study
}

\section{Amany Ibrahiem Ahmed Mohamed ${ }^{1 *}$, Hebatallah Mohammed El Far', Fatma Mohamed Saeed ${ }^{3}$ and Nehal Lotfy Abou Raya ${ }^{4}$}

${ }^{1}$ Masters Student, Faculty of Dentistry, Cairo University, Egypt

${ }^{2}$ Professor of Endodontics, Faculty of Dentistry, Cairo University, Egypt

${ }^{3}$ Lecturer of Endodontics, Faculty of Dentistry, Cairo University, Egypt

${ }^{4}$ Lecturer of Biomaterials, Faculty of Dentistry, Cairo University, Egypt

*Corresponding Author: Amany Ibrahiem Ahmed Mohamed, Masters Student, Faculty of Dentistry, Cairo University, Egypt.
Received: February 01, 2021

Published: February 26, 2021

(C) All rights are reserved by Amany Ibrahiem Ahmed Mohamed., et al.

\begin{abstract}
Aim of the Study: The aim of this study was to evaluate, setting time and solubility of Sure-Seal Root Bio-Ceramic sealer before and after mixing with Prednisolone powder.

Materials and Methods: Setting time test: sixteen Plaster of Paris molds (eight molds for each group) were soaked in distilled water for 24 hours then filled with the non-modified and modified sealer after addition of previously ground prednisolone powder to sealer and obtaining a homogenous mix. Setting time was calculated by using the Gilmore needle till the time that the needle probe fail to make indentation on the sealer's surface. Solubility test: Fourteen split Teflon molds (Seven molds for each group) were filled with non-modified and modified sealer as we mentioned before. Molds were placed between two pieces of wet cloth and two-glass slabs then kept in an incubator. Solubility was calculated as a percentage of mass loss at 24 hours, 7, 14, 28 days to determine the effect of time on the material solubility.

Results: The addition of prednisolone powder to Sure-Seal Root BC-Sealer caused a statistically significant increase in the setting time and the solubility of the sealer.

Conclusion: Addition of prednisolone powder to sure-seal root bio-ceramic sealer caused an increase in both the setting time and the solubility.
\end{abstract}

Keywords: Bio-ceramic Sealer; Setting Time; Solubility; Prednisolone

\section{Introduction}

Despite the recent advances in dental materials and techniques, dental pain especially endodontic pain remains a major problem in achieving a high quality patient care. It was reported that endodontic treatment is effective in reducing such pain, but the pain relief is rarely immediate and complete [1]. Endodontic pain can be managed during all phases of treatment: pre-operative, intra-operative and post-operative by using different pharmacological agents such as analgesics, anti-inflammatory drugs and corticosteroids. Inflammation of periapical tissue has been claimed to be source of this pain [2]. 
Corticosteroids are known to be effective drugs in reducing post-operative endodontic pain by working on the inflammatory process itself irrespective of the cause and the effect provided by them depends on their type and dose [3,4]. Prednisolone is a synthetic glucocorticoid. It's mode of action is by alternation of mRNA gene transcription, production of new proteins and has anti-phospholipase A2 activity, leading to the prevention of the synthesis of arachidonic acid products [2]. Prednisolone has superior pain reduction and the relatively higher efficacy of prednisolone is attributed to the less time it needs to diffuse across cell membrane and alter gene transcription [4].

Medicated sealers were introduced in the endodontic field as one of the methods used for local application of the drugs to increase its effectiveness and reducing its systemic side effect [5-8].

Endodontic sealers are used to form a coherent mass between the filling material and the root dentine. A new material (bio-ceramic sealer) has been developed and are characterized by an excellent sealing ability, antibacterial properties, biocompatibility and bioactivity as it provides a good tissue response if accidently extruded from the canal system and get into contact with the periapical tissues [10].

Calcium silicate based sealers are presented in two forms; the one component sealer (Ready-to-use) available in a premixed syringe with calibrated intra-canal tips and utilizes external water supply to set and the two component sealer with internal water supply [9]. Recently, a premixed injectable calcium silicate based sealer known as Sure Seal Root BC-Sealer was introduced into the market. As stated by the manufacturer, it utilizes moisture to initiate and complete its setting reaction, once setting occurs, the result is a chemical bonding with a void-free interface between the gutta-percha, sealer and radicular dentin. Its physicochemical properties have been the subject of considerable attention.

The properties of root canal sealers can be divided into the following categories: physicochemical, antimicrobial, and biological. When studying the ideal properties of a filling material it is possible to establish research parameters for the development of new products and to evaluate those already existing on the market, thus achieving better clinical results in clinical practice [11].

To our knowledge uptill now, there is no any previous studies which tried adding corticosteroids as an anti- inflammatory agent to root canal sealers. Therefore, Physical tests on pure (Sure-Seal Root bio-ceramic sealer) and combined with anti-inflammatory (Prednisolone modified Sure-Seal Root bio-ceramic sealer), were conducted to demonstrate if the addition of prednisolone to Sure-Seal Root bio-ceramic sealer would affect its physical properties.

\section{Materials and Methods}

Preparation of test group

Prednisolone powder 5mg (Solupred Oro tablets) was ground using agate mortar and pestle to avoid any impurities. After that, one gram Sure-Seal Root Bio-ceramic sealer was evacuated on a glass slab and mixed with $5 \mathrm{mg}$ pre- grinded prednisolone powder to attain 5\% concentration (https://www.drugs.com/dosage/ prednisone.html) [12]. Spatualtion was carried out on a glass slab by using a cement spatula until all the powder particles were adequately incorporated into the mixture

\section{Setting time}

The setting time of the sealers was determined according to ISO 6876:2012 [13]. Sure Seal Root bioceramic sealer was tested by dispensing the material into 4 Paris molds containing 4 openings with $10 \mathrm{~mm}$ diameter and $2 \mathrm{~mm}$ height. For bioceramic materials require moisture for setting, the Paris molds were first stored at $37 \mathrm{oC}$ in a water bath for 24 hours. The modified cement and the non-modified one were transferred into the corresponding molds $(\mathrm{n}=8)$ and the whole assemblies were stored in an incubator at 37 ${ }^{\circ} \mathrm{C}$ and $100 \%$ relative humidity. Gilmore needle with a mass of 100 gm and $2 \mathrm{~mm}$ active tip was lowered vertically onto the horizontal surface of the testing sample. The materials were tested every 10 minutes. Once partial failure of indentation was inspected the time was decreased gradually till reaching the setting time and indentations were inspected using a magnifying lens. The needle tip was cleaned before each time. The time from the start of mixing till the sealer completely set was calculated as the setting time

\section{Solubility}

The Solubility of the tested specimens was assessed according to the International Standards Organization (ISO) No.6876 (ISO 6876/2012) and the ANSI/ADA specification No.57 for "Materials used for permanent root canal sealer" [14]. Fourteen Specimens (7 for each group) were prepared using split Teflon molds having an internal diameter of $7.75 \mathrm{~mm}$ and a height of $1.5 \mathrm{~mm}$. Specimens were slightly modified as suggested by Carvalho-Junior., et al. 2007 [15]. 
The mold was filled to a slight excess with freshly mixed sealer. A nylon thread was placed inside the material, in order to suspend the samples in water However, For Sure-Seal BC sealer that require moisture for setting, two pieces of wet cloth were placed between the mold and the glass plates then the mold containing the specimens was pressed between them gently in such a way that the plate touched the entire mold in a uniform manner to ensure that the sealer material was spread uniformly throughout the mold; no air bubbles remained and to extrude any excess material, then the specimens were left in the incubator at $37^{\circ} \mathrm{C}$ and $>95 \%$ relative humidity for a period corresponding to three times

The samples were weighed 3 times using an electric balance. The average reading was recorded to 3 decimal places. The samples were placed into a Petri dish containing $7.5 \mathrm{~mL}$ of distilled water without any contact with inner surface and transferred into the incubator at $37^{\circ} \mathrm{C}$ and $>95 \%$ relative humidity in a tight sealed petri tube for different time intervals: 24 h, 7, 14 and 28 days respectively to test the solubility, At each end point. After incubation time, the Petri dishes were dried to a constant weight at $110 \pm 5^{\circ} \mathrm{C}$ and reweighted. The difference between the final mass and the initial mass of the Petri dish divided by the initial dry weight of the sample $\mathrm{x} 100$ correspond to the loss of mass of each specimen express as percentage of solubility. The solubility test was repeated after immersion in deionized water for 24 hours, 7, 14, 28 days by using the same method.

\section{Statistical analysis}

The results were tabulated and statistically analyzed. Data were presented as mean and standard deviation. Shapiro Wilk test was used for data normality and Independent $t$ test was used for analyzing data between the two groups. While paired-t test was used for data within the same group. Significance of the obtained results was judged at the $5 \%$ level.

\section{Results}

Regarding setting time

Prednisolone modified BC-sealer group (the intervention group) showed a statistically significant longer setting time than that of the Sure-Seal Root BC-sealer group (the control group).

Results of the comparison of the setting time between the Prednisolone modified BC-sealer group and Sure-Seal Root BC-sealer group.

\begin{tabular}{|l|c|c|c|}
\hline & Intervention Group & Control Group & P - Value \\
\hline Mean & 427.8 & 237.3 & \\
\hline SD & 1.4 & 5.6 & $<0.001$ \\
\hline
\end{tabular}

Table a

Regarding the solubility percentage between the two groups at different time intervals It was found that Prednisolone modified $\mathrm{BC}$-sealer (the intervention group) had a significantly higher solubility percentage values compared to Sure-Seal Root BC-sealer (the control group) over all the tested time intervals of 1 day ( $p<0.001$ ), 7 days $(\mathrm{p}=0.034), 14$ days $(\mathrm{p}<0.001)$ and 28 days $(\mathrm{p}=0.002)$.

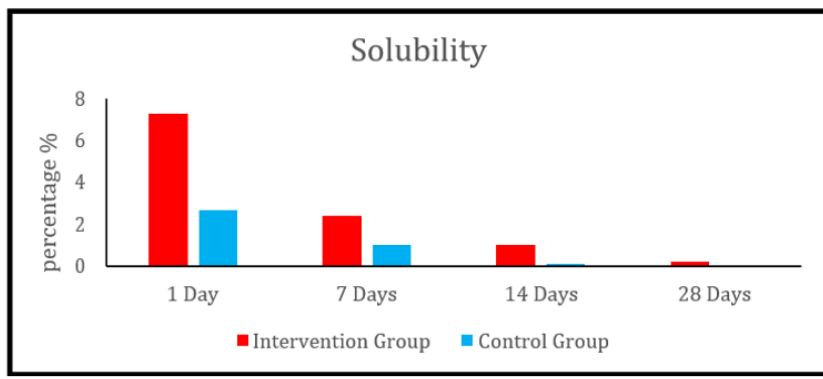

Figure 1

Bar chart representing the mean solubility percentage (\%) after $1,7,14$ and 28 days in the two groups.

Regarding the rate of solubility of Prednisolone modified BC-sealer group (the intervention group) samples decreased significantly over each time interval. The same behavior was spotted for Sure-Seal Root BC-sealer (the control group).

Line chart representing the rates of solubility at different time intervals within the two groups.

\section{Discussion}

With new endodontic sealers being successively developed by manufacturers, it has become important for the clinician to understand the sealer properties. Preliminary laboratory studies contribute to a better understanding of the clinical behavior and handling performance of endodontic sealers modification [11]. It is essenti- 


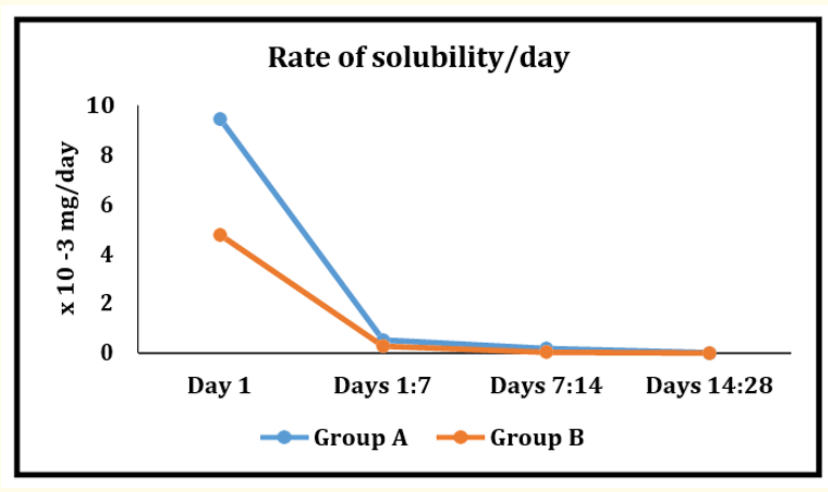

Figure 2

al to establish standardized methodologies to evaluate these properties in order to obtain reliable data and reproducible results from several studies. Therefore, the current study tested physical properties according to the International Standards Organization (ISO) No.6876 and ANSI/ADA specification no 57.

Prednisolone is characterized by higher efficacy in post-operative pain reduction [16]. The intra-canal use of Prednisolone with Sure-Seal Root BC-sealer could be considered the easiest, the most non-invasive way for drug administration and of limited circulating systemic effect as only a very small amount of medication is locally used for direct and immediate release to inflamed periapical tissue which result in rapid pain relief at the period during which most patients report feeling pain [17].

New bio-ceramic endodontic sealers provide an attempt to create sealer with ideal physicochemical and biological properties. Such endodontic sealer is Sure Seal Root BC-Sealer. Setting time test was measured because it is counted as a control test on the stable behavior of a material [18]. According to the manufacturers' sure-seal root sealers require moisture for setting. Therefore Plaster of Paris mold was soaked in distilled water for 24 hours before used.

The slight prolonged in vitro setting time of bioceramic sealers, in this study, could be explained by the absence of tissue fluids inside the dentinal tubules of the root canals which is considered the main source of continuous moistening during the hydration reacti- on $[19,20]$. Also, the setting time was different from that reported by the manufacturer. This discrepancy may be attributed to variations in the testing method used or may be a result of the unmetered moisture levels of the Paris molds used in experiment.

Prednisolone modified BC-sealer had a significantly higher setting time than the Sure-Seal Root BC-sealer and it may be attributed to incorporation of Prednisolone as a powder, which could interfere with the setting reaction of the sealer. The results are in agreement with Ruiz-Linares., et al. 2013, Shih., et al. 2014 and Arias-Moliz., et al. 2015 who found that different additives caused increase in the setting time of permanent root canal sealers [6,21,22]. However, the result was in contrast to Razmi., et al. 2010 who used amoxicillin antibiotic powder as an additive to sealer which caused a decrease in the setting time of the AH/26 resin sealer also the results were in contact with Weckwerth., et al. 2015 who stated that the addition of different antifungal drugs to sealers reduced the setting time of Fillapex $[7,23]$.

Solubility test was carried out to determine the suitability, clinical performance and durability of sealers. Solubility was measured according to ISO 6876/2012 and the ANSI/ADA specification No.57 $[13,14]$. The modified- split Teflon molds having an internal diameter of $7.75 \mathrm{~mm}$ and a height of $1.5 \mathrm{~mm}$ for the solubility test was used to allow an $85 \%$ reduction in the volume of necessary material when compared with the standard test sample without affecting the accuracy based on Carvalho-Junior., et al. 2007 [15].

The residue method was used as the loss of mass of each specimen (percentage of solubility) was calculated by the difference between the final mass and the initial mass of petri-dish divided by the initial dry mass of each specimen $* 100$ to exclude the weight of the sample itself because bioactive properties of calcium silicate sealers is characterized by the formation of deposits that adhere onto the specimen's surface which could interfere with the final mass of specimens. Also, the hydraulic nature of this material as water uptake may compensate for the dissolved material if measurements depend on the weight of specimens that may result in misleading solubility percentage [24-27].

Generally, the high solubility of bio-ceramic sealers could be the result of the hydrophilicity and nano-sized particles of sealer which increases the material surface area and allows more liquid molecules to ingress into the sealer over time [11,28-30]. In addi- 
tion, it could be due to exposing the specimens to large amounts of deionized water throughout the experimental period, the osmotic effect would have enhanced the solubility of sealer to a greater degree than might be found in any clinical situation (i.e. periapical tissue) [31].

In this study, the solubility of Sure-Seal Root BC-sealer was in agreement with ISO 6876:2012 and ANSI/ADA specification no. 57 in spite of representing the maximum permitted limit. This result was in line with previous studies [25,31,32]. However, in contrast with other studies by Borges., et al. 2012, Poggio., et al. 2017, Siboni., et al. 2017, Boyadzhieva., et al. 2017, Colombo., et al. 2018 and Zamparini., et al. 2018 that reported that BC sealer does not respect the ISO 6876 and ADA/ANSI specification No 57. The discrepancy between the results may be attributed to variation in the methods used to calculate solubility.

The results of the present study revealed that solubility percentage of prednisolone modified BC-sealer showed significantly higher levels compared to Sure-Seal Root BC-sealer at all tested time intervals. This may be related to its high initial setting time as stated by Zamparini., et al. 2018 or the possibility of incorporation of air during spatulation technique that might have led to voids entrapment. This porosity could have been occupied by water resulting in more dissolution of the immature matrix of prednisolone modified BC-sealer.

The solubility rate of prednisolone modified BC-sealer exhibited initial burst at first day that decreased significantly over time till reaching minimum or almost no solubility after 28 days. This high percentage of solubility at 24 hours could be considered as advantageous. Based on the pattern of post-operative pain and knowing that most of it occurs immediately after termination of root canal treatment [1]. On studying profoundly the percentage of residues that leached out from the prednisolone modified $\mathrm{BC}$-sealer, it may be assumed that it was equal to $7 \%$ which stood for the $2.6 \%$ that regularly leached out of the non-modified BC-sealer in additional to prednisolone (3.4\%). Therefore, the leaching out of most prednisolone in the first 24 hours may help alleviation of immediate postoperativepain.

The significant decline in the solubility percent by time in both groups can be explained on the basis of the depletion of the material due to proceeding of setting reaction that may be taking up to one month as stated by Donnermeyer., et al. 2018. Also, it may be speculated that the formation of crystals leads to a kind of self-sealing effect of sealer, because the precipitation is undergoing further growth and maturation and is becoming more stable on the long term which may lead to further decrease in solubility [30,32].

Furthermore, in the current study, the slight high solubility is desirable in order to allow the local dissolution of the medication into the periapical tissue. Moreover, slightly prolonged setting time and high solubility especially of calcium silicate containing sealers could be considered beneficial from biological point of view because the continuous release of calcium hydroxide and high alkalinity may enable this sealer to exhibit a more powerful antibacterial effect.

\section{Conclusion}

Within the limitations of this study, it could be concluded that, the setting time of both materials are within the same ranges for other BC-sealer. Also, In vitro testing of the setting time of the sealers may present a larger duration due to the difference in the nature of the surrounding media between the in-vitro and in clinical situation. Prednisolone modified BC-sealer exhibit high solubility levels compared to non-modified Sure-Seal Root BC-sealer.

\section{Conflict of Interest}

The authors deny any conflicts of interest in this study.

\section{Bibliography}

1. Suneelkumar Chinni., et al. "Effect of preoperative corticosteroids in patients with symptomatic pulpitis on postoperative pain after single-visit root canal treatment: a systematic review and meta- analysis". Journal of Endodontics 44.9 (2018): 1347-1354.

2. Praveen R., et al. "Comparative evaluation of premedication with ketorolac and prednisolone on postendodontic pain: a double-blind randomized controlled trial". Journal of Endodontics 43.5 (2017): 667-673.

3. Shamszadeh Sayna., et al. "Efficacy of corticosteroids on postoperative endodontic pain: a systematic review and meta-analysis". Journal of Endodontics 44.7 (2018): 1057-1065.

4. Nogueira Brenna ML., et al. "Is the use of dexamethasone effective in controlling pain associated with symptomatic irre- 
versible pulpitis? A systematic review". Journal of Endodontics 44.5 (2018): 703-710.

5. Baer J and Maki J. "In vitro evaluation of the antimicrobial effect of three endodontic sealers mixed with amoxicillin". Journal of Endodontics 36.7 (2010): 1170-1173.

6. Arias-Moliz Maria Teresa., et al. "The effect of benzalkonium chloride additions to AH Plus sealer. Antimicrobial, physical and chemical properties". Journal of Dentistry 43.7 (2015): 846-854.

7. Weckwerth Paulo Henrique., et al. "Effects of the association of antifungal drugs on the antimicrobial action of endodontic sealers". Brazilian Oral Research 29.1 (2015): 1-7.

8. Saghiri M A., et al. "The influence of antibiotics on the physical properties of endodontic cements". Giornale Italiano di Endodonzia 30.2 (2016): 89-95.

9. Donnermeyer, David, et al. "Endodontic sealers based on calcium silicates: a systematic review". Odontology 107.4 (2019): 421-436.

10. Lim Miyoung., et al. "Calcium silicate-based root canal sealers: a literature review". Restorative Dentistry and Endodontics 45.3 (2020).

11. Boyadzhieva, E., et al. "Setting time and solubility of premixed bioceramic root canal sealer when applicated with warm gutta percha obturation techniques". IOSR Journal of Dental and Medical Sciences 16 (2017): 125-129.

12. https://www.drugs.com/dosage/prednisone.html

13. International Organization for Standardization. International Standard ISO 6876:2012: Dental root canal sealing materials. Geneva: International Organization for Standardization (2012).

14. ANSI/ADA. "Specification no 57 endodontic sealing material". Chicago, USA: ADA Publishing (2000).

15. Carvalho-Junior Jacy R., et al. "Solubility and dimensional change after setting of root canal sealers: a proposal for smaller dimensions of test samples". Journal of Endodontics 33.9 (2007): 1110-1116.
16. Elkhadem A., et al. "The effect of preoperative oral administration of prednisolone on postoperative pain in patients with symptomatic irreversible pulpitis: a single-centre randomized controlled trial". International Endodontic Journal 51 (2018): e189-e196.

17. Iranmanesh Foad., et al. "Effect of corticosteroids on pain relief following root canal treatment: A systematic review". Iranian Endodontic Journal 12.2 (2017): 123.

18. Marín-Bauza Gabriela Alexandra., et al. "Physicochemical properties of endodontic sealers of different bases". Journal of Applied Oral Science 20.4 (2012): 455-461.

19. Lee Ju Kyung., et al. "Physicochemical properties of epoxy resin-based and bioceramic-based root canal sealers". Bioinorganic Chemistry and Applications 2017 (2017).

20. Abu Zeid Sawsan Taha., et al. "Setting reaction of new bioceramic root canal sealers". Spectroscopy Letters 51.8 (2018): 426-430.

21. Ruiz-Linares Matilde., et al. "Physical properties of AH Plus with chlorhexidine and cetrimide". Journal of Endodontics 39.12 (2013): 1611-1614.

22. Shih Yin-Hua., et al. "Evaluation physical characteristics and comparison antimicrobial and anti-inflammation potentials of dental root canal sealers containing hinokitiol in vitro". PloS one 9.6 (2014): e94941.

23. Razm Hasan., et al. "Comparison of AH26 physicochemical properties with two AH26/antibiotic combinations". Iranian Endodontic Journal 5.1 (2010): 6.

24. Abu Zeid S T H and A A Y Mokeem Saleh. "Solubility, pH Changes and releasing elements of different bioceramic and mineral trioxide aggregate root canal sealers comparative study". Journal of Trauma and Treatment 4.249 (2015): 2167- 1222.

25. Prüllage Raquel-Kathrin., et al. "Material properties of a tricalcium silicate-containing, a mineral trioxide aggregate-containing, and an epoxy resin-based root canal sealer". Journal of Endodontics 42.12 (2016): 1784-1788.

26. Poggio Claudio., et al. "Solubility and $\mathrm{pH}$ of bioceramic root canal sealers: a comparative study". Journal of Clinical and Experimental Dentistry 9.10 (2017): e1189. 
27. Tanomaru-Filho Mario., et al. "Physicochemical properties and volumetric change of silicone/bioactive glass and calcium silicate-based endodontic sealers". Journal of Endodontics 43.12 (2017): 2097-2101.

28. Al-Haddad Afaf and Zeti A Che Ab Aziz. "Bioceramic-based root canal sealers: a review". International Journal of Biomaterials 2016 (2016).

29. Mendes Aline Teixeira., et al. "Evaluation of physicochemical properties of new calcium silicate-based sealer". Brazilian Dental Journal 29.6 (2018): 536-540.

30. Donnermeyer D., et al. "The push-out bond strength of calcium silicate-based endodontic sealers". Head and Face Medicine 14.1 (2018): 1-7.

31. Ersahan Seyda and Cumhur Aydin. "Solubility and apical sealing characteristics of a new calcium silicate-based root canal sealer in comparison to calcium hydroxide-, methacrylate resin-and epoxy resin-based sealers". Acta Odontologica Scandinavica 71.3-4 (2013): 857-862.

32. Urban Kent, et al. "Solubility and $\mathrm{pH}$ value of 3 different root canal sealers: a long-term investigation". Journal of Endodontics 44.11 (2018): 1736-1740.

33. Borges R P., et al. "Changes in the surface of four calcium silicate-containing endodontic materials and an epoxy resin-based sealer after a solubility test". International Endodontic Journal 45.5 (2012): 419-428.

34. Siboni F., et al. "Properties of BioRoot RCS, a tricalcium silicate endodontic sealer modified with povidone and polycarboxylate". International Endodontic Journal 50 (2017): e120-e136.

35. Colombo Marco., et al. "Biological and physico-chemical properties of new root canal sealers". Journal of Clinical and Experimental Dentistry 10.2 (2018): e120.

36. Zamparini Fausto., et al. "Properties of calcium silicate-monobasic calcium phosphate materials for endodontics containing tantalum pentoxide and zirconium oxide". Clinical Oral Investigations 23.1 (2019): 445-457.

\section{Assets from publication with us}

- Prompt Acknowledgement after receiving the article

- Thorough Double blinded peer review

- Rapid Publication

- Issue of Publication Certificate

- High visibility of your Published work

Website: www.actascientific.com/

Submit Article: www.actascientific.com/submission.php

Email us: editor@actascientific.com

Contact us: +919182824667 